\title{
Dichtung und Daten. Programmcode und das Werk des österreichischen Künstlers Jörg Piringer
}

\section{Poetry and Data. Program Code and the Work of the Austrian Artist Jörg Piringer}

\section{Zdeněk Pecka}

\begin{abstract}
The difficult distinction between reality and virtuality, between fact and fiction, user and read$\mathrm{er}$, and between author and narrator becomes the inseparable quality of digital literature. Using the example of "Datenpoesie" (2018) by the Austrian artist Jörg Piringer, the article examines the boundary between an electronic device as a medium competing with the traditional carrier such as books, and a technological format that already has effects on the genres.
\end{abstract}

\section{Keywords}

digital poetry; code poetry; literature and technology; digital turn; media; new media; Jörg Piringer; Austrian Literature 
Im vorliegenden Beitrag wird das Werk des gegenwärtigen österreichischen Künstlers Jörg Piringer aus der Perspektive der literarischen Innovation untersucht. Der Autor meldet sich zum Erbe der österreichischen Nachkriegsavantgarde und bewegt sich in der gegenwärtigen Szene der elektronischen Kunst. Sein relativ wenig bekanntes Schaffen wird in den Kontext der gegenwärtigen digitalen Poesie gesetzt, es werden sein Blick auf die digital entstehende Literatur vermittelt und schließlich seine in Druckform erschienene Sammlung datenpoesie (2018) behandelt.

\section{Code Poetry}

Die digitale Wende brachte in den letzten Jahrzehnten einen Umbruch in vielen Bereichen des Lebens sowie des gesellschaftlichen Verkehrs und damit auch in den Geisteswissenschaften und in der Literaturwissenschaft. In ihrem Bereich kann man sich methodologisch auf die ,traditionelle' Literaturtheorie, auf die Literatursoziologie Pierre Bourdieus $^{1}$ sowie auf die Digital Humanities ${ }^{2}$ stützen. Mit der Bezeichnung der Digital Humanities sind immer noch terminologische Unklarheiten verbunden, die das noch junge Alter der Disziplin widerspiegeln. Die Erscheinung des Computers in der zweiten Hälfte des 20. Jahrhunderts und die damit verbundenen quantitativen Ansätze in den Geisteswissenschaften brachten den Terminus ,Humanities Computing“, der mit der Verbreitung des Internets und der Vermehrung von computerbasierten mobilen Geräten sich um die Jahrtausendwende in ,Digital Humanities' transformierte. Gleichzeitig bestätigte sich der interdisziplinäre Charakter des Wissenschaftsgebiets, denn neben den mit computergestützten Methoden längst vertrauten Disziplinen aus dem Bereich der exakten Wissenschaften begannen sich teilweise auch die Kulturwissenschaften wie Geschichte oder Literaturwissenschaft auf computergesteurte Datenanalyse basierte Methoden zu stützen.

Die Ursprünge der elektronisch bedingten Literatur muss man bei der konkreten Poesie und der Stuttgarter Gruppe suchen. Diese Gruppierung um Max Bense versuchte schon Ende der 50er, Anfang der 60er Jahre mittels Computer literarische Texte $\mathrm{zu}$ generieren. Ihre Experimente mit den damaligen Medien und elektronischen Aufschreibsystemen fanden ein bedeutsames Echo in vielen Ländern, u. a. auch unter den international bedeutenden Autoren konkreter und visueller Poesie in der damaligen Tschechoslowakei wie Václav Havel, Josef Hiršal, Bohumila Grögerová oder Ladislav Novák. ${ }^{3}$ Die digitale Poesie hat sich in den 90er Jahren als eigenständige Kunst etabliert und hat sich aus der Netzliteratur zur autonomen Richtung in der digitalen Kunst entwickelt. Die Digitalität und der Umgang mit Sprache als einem Instrument und Material zugleich sind die Grundvoraussetzungen der digitalen Poesie. Das Arbeitswerkzeug bildet der Computer, was auch die Selbstbezogenheit der Kunst bedingt, deren Autonomie

$1 \quad$ Vgl. Bourdieu 1982 und Bourdieu 1999.

2 Vgl. Reiche 2014.

3 Vgl. Döhl 1997 oder Suter 2012. 
durch die computergesteuerten Prozesse und Programmstrukturen ermöglicht wird. Diesen Selbstbezug bestimmen einige wesentliche Eigenschaften der digitalen Poesie wie Vernetzung und Interaktivität, Dynamik und Hypermedialität. Die Vernetzung der modernen Literatur erfolgt durch das Internet und die vernetzten Computer, wobei die Interaktivität zwischen Menschen und dem Computer eingestellt ist. Der Prozess der Entstehung wird unter der Bedingung der Transfugalität - Vergänglichkeit der digitalen und der Netzkunst - zum eigentlichen Zweck der digitalen Poesie und während der Produktion begleiten ihn unterschiedliche dynamische Animationen und Bewegungen, die sich in unterschiedlichen digitalen Medienformaten widerspiegeln, deren ,Endprodukte' neben Text auch Bild-, Video- oder Audiodateien bilden. Diese entstehen durch die tiefen Textstrukturen in den Programmierebenen der Soft- und Hardware des Mediums und die Vernetzung und die Interaktion als Grundelemente der Prozessualität der digitalen Poesie werden durch den Dialog zwischen Benutzer und Computer entwickelt. Eine der allgemein anerkannten zusammenfassenden Definitionen lautet:

„Der Begriff , digitale Poesie“ lässt sich [...] künstlerischen Projekten zuschreiben, die sich mit den medialen Veränderungen von Sprache und sprachbasierter Kommunikation in Computer und digitalen Netzwerken auseinandersetzen ${ }^{\star 4}$

Codework oder ,Program Code Poetry“ hat sich in den neunziger Jahren als Unterhaltung von Programmierern und Cyber-Autoren etabliert, die mittels eines Programmcode versuchten, auch literarische Qualitäten zu erreichen. Codework arbeitet mit der Schnittstelle, der Grenze zwischen der realen und virtuellen Welt vernetzter und programmierbarer Medien. Die Texte referieren auf die digitale Basis und bezeichnen die Beziehung zwischen dem Code im Programm und der Interface auf der Oberfläche. Unbezweifelbar aber ist, dass

„[...] Codeworks eine besonders hohe (und bei Künstlern seltene) Kompetenz in Sachen Internettechnologien und Computersprachen voraussetzen. Wichtig ist zudem eine enorme Affinität zu diesen basalen, betriebssystemnahen Sphären von Code. “5

Der Programmcode ist (ziemlich unsichtbar) ein so präsentes Element in der Gegenwart, dass es nicht überraschend ist, dass er sowohl auf der sprachlichen Ebene als Inspirationsquelle erscheint, als auch im thematischen oder motivischen Bereich als eine beunruhigende Aktualität zur Äußerung kommt. Auch die jüngere Generation von Künstlern widmet sich in der Gegenwart dem Code als literarischem Stoff. Im deutschsprachigen Raum ist es der Berliner, bzw. Basler Dichter und Wissenschaftler Hannes Bajohr, der häufig über die Code Poetry publiziert und der im Jahr 2016 die Anthologie zur Code Poetry und der konzeptuellen Literatur ${ }^{6}$ unter dem Titel Code und Konzept

4 Block, Friedrich 2004, S. 12.

5 Hartling, Florian 2009, S. 281.

6 Vgl. Goldsmith 2014. 
herausgab. Im theoretischen Programmkapitel formuliert Bajohr die Bemühung, die konzeptuelle Literatur und die Codeliteratur als zwei verwandte Richtungen in der gegenwärtigen experimentellen Literatur darzustellen. „Ihre Mittel und Methoden ähneln sich häufig, ihre Referenzgrößen und ihre Übersetzungen, was den Status von Text, Autor und Performance in der Literatur der Gegenwart angeht, sind oft dieselben. " behauptet Bajohr. Er meldet sich zur Tradition des konzeptuellen Schreibens, wie es die Autoren Christian Bök, Vanessa Place, Kenneth Goldsmith, oder Craig Dworkin repräsentieren, die laut Bajohr sowieso die historischen Avantgarden und Dada mit modernen Mitteln fortsetzen.

\section{Jörg Piringer}

Jörg Piringer ist ein in Wien lebender Künstler und Wissenschaftler, der in den Bereichen Lautpoesie, visuelle Poesie, Klanginstallationen, elektronische Musik oder interaktive poetische Systeme aktiv ist. Er arbeitet dabei jedoch oft auch mit dem Medium Video und führt Live-Performances in Clubs oder Festivals auf. Einige Tools, die für seine Veranstaltungen entwickelt worden sind, bietet Piringer auf seiner Homepage ${ }^{8}$ als Programme oder als Apps für das Smartphone iPhone oder sogar für die digitale Armbanduhr Apple Watch. ${ }^{9}$ Der studierte Informatiker betrachtet einen (literarischen) Text oder ein textbasiertes Kunstwerk nicht nur als eine fertige Form, sondern bemüht sich in seinen Performances auch um einen zum Ergebnis führenden kreativen Prozess. In seinem Fall bedeutet es einerseits die Programmierarbeit, durch die erst Instrumente entstehen, mit denen man das Kunstwerk formiert. Andererseits ist es dann der schöpferische Fortgang, der seine digitalen Installationen begleitet. Jörg Piringer ist ein sehr produktiver Autor, dessen Wirkung nicht nur durch seine Werke, sondern auch durch seine Druckpublikationen und z.B. popularisierende Interviews erfolgt, die zur Etablierung der Codeliteratur als souveräner Richtung in der gegenwärtigen avantgardistischen Literatur beitragen sollen. Der Autor ist ihr repräsentativer Darsteller, indem er seine literarischen Werke durch den auf Computer ausgeführten Programmcode produziert. Die Codeliteratur basiert auf einem formalen Konzept, das durch Algorithmen der Software und Computeroperationen realisiert wird. Als Beispiel lässt sich die Codezeile „\$wurm = (\$apfel>0) ? $1: 0 ; “ 10$ anführen, die im Textcode genau das erzählt, was die berühmte Apfelanimation ${ }^{11}$ zeigt: „Ist der Apfel größer Null, is(s)t der Wurm. Ansonsten is(s)t er nicht."12 Es handelt sich um ein ganz einfaches ,Codework', das einerseits auch einem ganz wenig kompetenten Rezipienten verständlich ist, andererseits jedoch

7 Bajohr 2016, S. 7.

8 Vgl. http://joerg.Piringer.net (zuletzt geprüft am 15. 3. 2021).

9 Vgl. Piringer 2016, S. 241-246.

10 Auer 2015.

11 Auer 1997.

12 Auer [Anm. 7]. 
einen vom Computer ausführbaren Code in der Skriptsprache PHP darstellt. Dadurch entspricht der experimentelle Charakter dieser Literatur dem gegenwärtigen künstlerischen Schaffen im Zeitalter der fortschreitenden ,Digitalität'. Der deutsche Digitalkünstler Hannes Bajohr schreibt in diesem Zusammenhang:

\begin{abstract}
„Nimmt man die Prävalenz des Begriffes als Diskursmasse gerade in seiner Unbestimmtheit ernst, dann bezeichnet »digital « vielleicht am ehesten eine kulturelle Stimmung, die zwar ein technisches Fundament voraussetzt, aber vor allem davon abhängt, wie bewusst sie sich dieses Fundaments ist. Digitale Literatur wäre dann mehr als bloß digital produzierte, sondern solche, die dieses Wirklichkeitsverständnis selbst zeigt, anstatt es nur zu sagen [...].“13
\end{abstract}

Thematisch widmet sich Piringer hauptsächlich der Digitalisierung und ihren Auswirkungen auf das gegenwärtige Wahrnehmen der Privatsphäre, des Datenschutzes oder der künstlichen Intelligenz. Dabei spielt die ,Codekompetenz', also das Können von hoch formalisierten Programmiersprachen eine entscheidende Rolle. Nach Piringers Worten müssen sich Informatik und Literatur nicht ausschließen. „Man schreibt, nur werden die Texte eben nicht von Menschen gelesen, sondern von Maschinen. "14 Die Arbeit eines Programmierers ist im Prinzip „[...] eine poetische Arbeit. Du kreierst Texte, und die führen etwas aus. Genauso wie Lyrik im Kopf des Lesers etwas ausführt. ${ }^{\text {15 }}$ Die Technologie muss laut Piringer nicht unbedingt der Zentralpunkt der neuen Poesie sein, sondern sicher eine der Gegenwart entsprechende Inspirationsquelle. „Man kann nicht wirklich hergehen und dadaistische Schriftbilder wie im Jahr 1914 machen." ${ }^{16}$ In dem oben zitierten Interview für Der Standard meldet sich Piringer v. a. zum Erbe der europäischen literarischen Avantgarde und bekennt sich zur Inspirationsquelle für seine Werke u.a. bei der Wiener Gruppe:

„Ich habe irrsinnig viel gelernt von der Wiener Gruppe und ihrer Beschäftigung mit der Sprache als Material - beispielsweise von Gerhard Rühm. Im Gegensatz dazu arbeite ich aber viel mit Akustik, verwende Samples aus dem alltäglichen Leben und verändere bereits existierendes Material. “17

Gleichzeitig ist sich Piringer der Tradition der experimentellen elektronischen Musik, bewusst, die im deutschsprachigen Raum z. B. Karlheinz Stockhausen prägte, und in einer Sendung des SWR2 aus dem Jahr 2011 wird noch Henri Chopin als eine Inspirationsquelle genannt:

13 Bajohr [Anm. 2], S. 14.

14 Piringer 2013.

15 Piringer [Anm. 7].

16 Piringer [Anm. 7].

17 Thalmair 2009. 
„Sprecherin: [...] Henri Chopin setzte den ganzen menschlichen Körper als Instrument ein. [...] Jörg Piringer benutzt wie Henri Chopin seine Stimme als Klangkörper. Chopins Werkzeug war das Tonbandgerät, Jörg Piringer experimentiert mit dem Computer. Er möchte darauf aufmerksam machen, dass die Sprache nicht nur einen Sinn, sondern auch Klang und Emotionen transportiert." 18

Cara Wuchold, die Sprecherin der Sendung, die neben Jörg Piringer auch den digitalen Avantgardisten Johannes Auer als Gast begrüßte, betont die Sprache, die ähnlich wie bei der Wiener Gruppe oder im Werk von Henri Chopin, das Basismaterial seines Schaffens bildet. Jedoch arbeitet Piringer mit aktuellen technischen und technologischen Produktionstechniken, von denen der Computer, der Quellcode, also ein in einer Programmiersprache lesbarer Text, und die Benutzeroberfläche die zentrale Position einnehmen. Piringer als Programmierer bleibt in der Position des Autors, der durch die Gestaltung der Software quasi den Plot und die Struktur seiner Narration gestaltet. Die Teilnehmer seiner Performances befinden sich dann in der Rolle der Leser und Rezipienten. Die Vorgangsweise des Autors kann anschaulicher werden, wenn noch seine Beschreibung des Projekts Alphabet (2010) angeführt wird: ${ }^{19}$

\begin{abstract}
„Also das ist eigentlich eine Performance, die ich mach', wo ich live Text-Video generiere und gleichzeitig den Sound, also mit meiner eigenen Stimme, und wo sich das irgendwie mischt. Wo ich z. B. einen Buchstaben, der auch am Bildschirm oder am Projektor zu sehen ist, mit einem Klang belege, und der dann mit anderen Elementen, wiederum Buchstaben, interagiert und die sozusagen zusammen einerseits das Video machen, aber auch den Sound. Also auch der Sound a " ndert sich dadurch, dass z. B. ein ,A“ mit einem ,B“ zusammensto “ Bt, und gleichzeitig a" ndern sich auch die Bewegungen von diesen beiden. "20
\end{abstract}

Es ist Piringers übliche Arbeitsmethode, dass er Kompositionen aus Text und Ton erzeugt, deren typische Eigenschaften Instabilität und Vergänglichkeit sind. In der Kombination von Buchstaben oder Textfragmenten und Klang entsteht eine augenblickliche Interaktion, durch die mittels weiterer computergesteuerter Modulation Bild und Ton zum Produkt der Performance werden. Der Autor bewegt sich dabei auch nahe der gegenwärtigen elektronischen Musik, dem Musik-Sampling und nicht zuletzt der Computerspiele: „Das ist die narrative Form, die dem Computer vermutlich am ehesten entspricht." 21

Jörg Piringer ist kein Angehöriger einer breiteren Strömung. Auch im internationalen Vergleich gibt es relativ wenige vergleichbare Künstler, die sich so systematisch und langfristig der Code Poetry widmen würden. Günter Vallaster erinnert an den Band

18 Wuchold 2011.

19 Vgl. Piringer, 2010.

20 Wuchold 2011.

21 Thalmair 2009. 
Hannes Bajohrs Code und Konzept, wenn er Jörg Piringer in seiner Rezension zu datenpoesie in eine Gesellschaft platziert, die

„[...] vergleichsweise klein [ist], aber sehr aktiv und miteinander vernetzt, genannt seien exemplarisch noch Nick Montfort, der am Massachusetts Institute of Technology (MIT) Digital Media lehrt, der Berliner Hannes Bajohr, der digitale Lyrik verfasst, in der Slowakei beschäftigen sich Zuzana Husárová und Richard Kitta mit elektronischer Literatur.“22

Für frühe Werke Piringers waren eigenhändig erzeugte Geräte typisch, die mit einer Computereinheit verbunden waren und einen Text modulierten, wie z.B. beim Objekt fms.$^{23}$ Es handelte sich um ein Gerät mit Batterien, Verstärker und Lautsprecher, in dem das Lautgedicht Raoul Hausmans $F M S B W^{24}$ aufgenommen wurde. Wurde das kleine Gerät bewegt, spielte dieses die Aufnahme in modulierte Weise je nach der Bewegung nach. In der Performance Frikativ ${ }^{25}$ aus dem Jahr 2007 wird die Stimme des Künstlers zu seinem Instrument. Durch die Modulation im Computer entstehen gestörte Laute, die sich an der Leinwand als abstrakte visuelle Textkompositionen widerspiegeln. Piringer hält den Computer für ein ,literarisches Instrument‘ und die Sprache als die Schnittstelle zwischen Computerwissenschaft und Literatur:

\begin{abstract}
„Mit Sicherheit treffen sich die beiden im Material, also in der Sprache. Und - das mag jetzt vielleicht etwas komisch klingen - im Schreiben. In beiden Fällen, also sowohl in der Literatur als auch in der Informatik gibt es einen Autor und einen Leser. Beim Buch dechiffriert der Mensch den Text, beim Programmcode gibt es noch eine zusätzliche Ebene. In beiden Fällen muss der jeweilige Text aber zuerst interpretiert werden [...] Der Computer ist an sich ein literarisches Medium. Grafik und Sound, so wie wir das heute kennen, gibt es ja noch nicht so lange. [...] ich finde, man kann mit einem Text viel mehr sagen, als mit einem Bild - Text ist ein mächtiges Instrument. “26
\end{abstract}

\title{
3 datenpoesie
}

Im 2015 veröffentlichten Artikel was wird literatur? was wird poesie?27 denkt Jörg Piringer nicht nur über seine Arbeit und ihre Kontexte nach, wobei der Zentralbegriff seiner Überlegungen ,Poesie‘ lautet. Gleich in den ersten Sätzen nimmt Piringer den Begriff ,Poesie‘ aus dem Zusammenhang mit ,Literatur' heraus und erklärt sie zu einer „[...] kunstform, die den einschränkungen, die der literatur für gewöhnlich zugemutet

\footnotetext{
22 Vallaster 2018.

23 Piringer 2006.

24 Vgl. Hausmann 1972, S. 76.

25 Piringer 2009.

26 Thalmair [Anm. 13].

27 Piringer 2015.
} 
werden, nicht unterliegt." ${ }^{28}$ Zugleich grenzt sich der Verfasser von den Verwandlungen der Literatur in neuen digitalen Medien als prägendem Thema ab. ,Poesie‘ ist für Piringer eine von (nicht nur) literarischen Kategorien unabhängige Kunstform, die existenziell mit dem Experimentieren verbunden ist und in der alles erlaubt ist. Sie kann sich nicht nur auf die Buchform einer Gedichtsammlung beschränken, sondern

„[...] ist schon jetzt performance. ist schon jetzt bildende kunst. ist jetzt visuelle poesie. ist lautpoesie. ist konzeptuelle poesie. ist poesieskulptur. ist generative poesie. ist poesieapps. ist softwarepoesie. ist poesiefilm. ist filmpoesie. ist kombinatorische poesie. polypoetry. haptische poesie. spampoetry. samplepoesie. flarf. lettristische poesie. schreibmaschinenpoesie. tachygraphie. hypergraphie. poesiehardware. scotch art. e-poetry. digitale poesie. netzpoesie..." ${ }^{29}$

Auf die Frage des Grazer Literaturhaus-Dossiers: Was wird Literatur antwortet Piringer, dass alles, was man sich mit der technologischen Zukunft von Literatur vorstellen könne, sei die wahre Poesie jetzt schon längst. Die Zukunft wird laut dem Künstler nicht in neuen Publikationsformen oder neuen Fassungen des Urheberrechtes stattfinden. Die düstere Zukunft kennzeichnet der Rücktritt des Menschen aus der literarischen Kommunikation. „es ist die digitale sprachtechnologie der konzerne, die die zukunft der poesie und der sprache im allgemeinen verändern wird. " ${ }^{30}$ Sowohl auf der formalen Ebene der Programmiersprachen, als auch in der als menschlich vorgetäuschten Textverarbeitung werden, so Piringer, Maschinen die lesbare Kommunikation besorgen. Es wird die Aufgabe der Dichter sein, dieses maschinelle Schaffen zu stören, umzuprogrammieren und zu hacken. Gleichzeitig findet die Poesie wahrscheinlich neue Inspirationsquellen in neuen Bereichen wie Desinformation, Welt der Geheimdienste und ihrer Algorithmen, bio- und neuronale Technologien, Virtual Reality und Daten. Piringer schließt seine Überlegung mit Worten eines Mitglieds der Wiener Gruppe, zu dessen Tradition er sich offen meldet, ab:

„was aber bleibt und bleiben muss, ist der kern der poesie als sprachbasierte reflexion von wirklichkeit und vorstellung. oder wie es H.C. Artmann formuliert:

Der vollzogene poetische Act, in unserer Erinnerung aufgezeichnet, ist einer der wenigen Reichtümer, die wir tatsächlich unentreissbar mit uns tragen können. "31

Somit handelt es sich zwar um einen nicht gerade umfangreichen, aber doch programmatischen Text von Jörg Piringer, in dem er seine Themen und sein Nachdenken über Literatur und Kunst andeutet, und der auch die Orientierung seines späteren Schaffens im Bereich der Kunst und ,Poesie‘ zeigt. Signifikant ist in diesem Sinne die Diskussion

28 Piringer [Anm. 20].

29 Piringer [Anm. 20].

30 Piringer [Anm. 20].

31 Piringer [Anm. 20], H. C. Artmann zitiert nach Acht-Punkte-Proklamation des poetischen Actes von H.C. Artmann, in: Reichert, Klaus (Hg.): The Best of H.C. Artmann, Frankfurt am Main: Suhrkamp, Jahr und Seite nicht angegeben. 
der Jury über den Text $k u z u s h i^{32}$ von Jörg Piringer während der Verleihung des Bachmann-Preises für das Jahr 2020, wie sie der österreichische staatliche Sender ORF dokumentiert hat:

„Die Jury war sich nicht einig, ob es sich um einen Monolog eines frustrierten Internetentwicklers handelt oder um einen von einer Maschine erstellen Text, in dem es um die Kreativität der künstlichen Intelligenz gehe. “33

Allgemeingültig für diese Art der experimentellen Poesie scheint jedoch die Position des Jurymitglieds Philipp Tingler zu sein: „Er hätte sich gewünscht, dass er eine Auseinandersetzung mit künstlicher Intelligenz und Kreativität leiste. “34

Im Jahr 2018 gab Jörg Piringer seine Sammlung datenpoesie ${ }^{35}$ heraus, in der Texte aus 20 Jahren der künstlerischen Tätigkeit des Autors gesammelt worden sind. Es handelt sich um einen Band, der auf alle Formen der Arbeit mit den digitalen Medien verzichtet und nur daraus entstandene Texte und Textmaterial präsentiert. Es handelt sich jedoch um keinen Katalog der bisherigen Projekte sowie um keine Dokumentation des schöpferischen Verfahrens Piringers, gleichzeitig werden seine literarischen Ambitionen in dem Band nicht versteckt. Prozesse, die zu den Ergebnissen in Form von Gedichten und visuellen Konstellationen führten, werden in dem einleitenden Kapitel und im abschließenden Kommentar erläutert. Die Gestaltung des Bandes ist traditionell und zeugt davon, dass Piringer, der sich bisher konzeptuell dem Video, Ton und interaktiven Umgebungen widmete, sich diesmal mit dem Medium Buch und mit allem, was dazu gehört, auseinandersetzen wollte. Der Umschlag und seine (typo)grafische Bearbeitung spielen mit dem Titel im Geiste der konkreten Poesie: „da daten datenpoesie poesie es sie“ und deuten an, dass durch mechanische Zerlegung der Sprache in Bausteine, die weiter und neu gestaltet werden, die Möglichkeiten einer durch Technologie gesteuerten kreativen Dichterarbeit untersucht werden.

Wie es in den Texten von Piringer schon die Norm ist, wird auch in datenpoesie keine Groß- und Kleinschreibung unterschieden. Der Inhalt wird durch eine Einleitung eröffnet, die „Programm“36 heißt und laut Annalena Stabauer vom Wiener Kunstverein Alte Schmiede „[...] nicht ganz unironisch [... “37 gemeint sein kann. In diesem ,Programm“ formuliert der Autor die Voraussetzung, dass es große Korporationen mit ihren automatischen digitalen Systemen sein werden, die in naher Zukunft den geläufigen Umgang mit der menschlichen Sprache also auch mit der Literatur übernehmen. Der Band als eine repräsentative Ausgabe der durch unterschiedliche maschinell und digital gesteuerte Prozesse entstandene Texte „[...] lotet jene sprachtechnologien aus, die in zukunft

32 Piringer 2020.

33 ORF 2020.

34 ORF [Anm. 30].

35 Piringer 2018.

36 Piringer [Anm. 27], S. 6.

37 Stabauer 2019, S. 7. 
unsere interaktion mit digitalen systemen bestimmen werden. "38 Jörg Piringer verwendet dazu, wie er selbst schreibt,

„[... [ methoden der künstlerischen Forschung und der explorativen programmierung, die im gegensatz zur wissenschaftlichen forschung und zur ingenieursmässigen informatik den subjektiven aspekt, den künstlerischen wert und den persönlichen erkenntnisgewinn betonen. ${ }^{\text {"39 }}$

$\mathrm{Zu}$ den Grundwerkzeugen, die Piringer zu seiner Dichtung benutzt, gehören einerseits die auch in der gegenwärtigen globalisierten Wirtschaft eingesetzten künstlichen neuronalen Netze, automatische Übersetzungssysteme und generative Grammatiken, andererseits Vorgangsweisen aus dem Bereich der Biologie und Physik. ${ }^{40}$ Piringer beendet den programmatischen Teil des Buches mit folgenden Worten: „die vorliegenden texte und textbilder reflektieren diese strukturen und algorithmen, die unsere kommunikation und weltwahrnehmung zunehmend beeinflussen, und bringen deren materialität an die oberfläche." ${ }^{41}$ In der abschließenden methodologischen Erläuterung und Erklärung mit Kommentar und Glossar macht Piringer deutlich, dass ein großer und auf den ersten Blick unsichtbarer Teil des Werkes in Programmiersprachen geschrieben ist, was einen anderen Zugang des Lesers zu den vorgebrachten Texten voraussetzt. Einerseits kann man auf der Oberfläche Texte lesen, die eine spannende Fortsetzung der avantgardistischen Poesie des 20. Jahrhunderts darstellen, andererseits soll man aber auch die Konzepte und Methoden hinter den Texten verfolgen und mit anderen Verfahren der gegenwärtigen konzeptuellen und performativen Kunst in Kontext setzen.

Im Band selbst befinden sich in den Kapiteln „vers“, „frage und antwort“, „suchtext“, „fließtext“, „anordnung“ und „paratext" geordnete Texte und Grafiken, die in ihrer Genese unterschiedlichen Prozessen der digitalen Kodierung und Dekodierung, ggf. maschinellen Übersetzung ausgeliefert wurden. Man beobachtet den experimentellen, manchmal spielerischen Charakter dieser Verfahren, der sich in der literarischen und grafischen Qualität der Texte widergespiegelt hat. Der Qualität ist jedoch ihre algorithmische Grundlage nicht wegzudenken, sondern sie wurde zum Bestandteil dieser Qualität. Im Rahmen der experimentellen Literatur gehört der Band Jörg Piringers zu Werken, die die Kategorien der avantgardistischen, konkreten und konzeptuellen Poesie oder des „Dada 2.0“42 gerade durch eine repräsentative Buchpublikation legitimieren und zu einem neuen Niveau bewegen.

38 Piringer [Anm. 27], S. 6.

39 Piringer [Anm. 27], S. 6f.

40 Vgl. Piringer [Anm. 27], S. 7.

41 Piringer [Anm. 27].

42 Vgl. Vallaster [Anm. 14]. 


\section{Fazit}

Jörg Piringer bemüht sich um die Fortsetzung der mittlerweile traditionellen Avantgarde mit neuen und aktuellen Mitteln sowie Themen. Gerade in den Attributen der digitalen Poesie wie Vernetzung und Interaktivität, Dynamik und Hypermedialität bemüht er sich um eine Anknüpfung und Aktualisierung. Sowohl die Vernetzung als auch Interaktivität bestehen bei seinen Auftritten gleichermaßen unter Leuten und Computern und die Transfugalität wird zu einer Grundvoraussetzung sowohl seiner Veranstaltungen, als auch der digitalen Poesie allgemein. Somit ähneln die Performances, bei denen der unmittelbare Kontakt mit dem Publikum eine wesentliche Rolle spielt, z. B. den Cabarets der Wiener Gruppe. Piringer, Programmierer und Dichter zugleich, gestaltet durch das Beherrschen der Software quasi den Plot und die Struktur seiner Narration, deren Ergebnis eher zum Experiment als zur Provokation wird. Durch die Herausgabe der Sammlung datenpoesie bestätigt der Autor sein Schaffen als finale Textmaterie, die einerseits als Dokumentation dauerhaft erhalten bleibt, deren innovative Entstehungsgeschichte jedoch andererseits zum großen Teil verloren geht.

\section{Quellen und Literatur}

Auer, Johannes: Vom Web 1.0 zum Postinternet. (Fast) alles über Netzliteratur in 3 1/2 Kapiteln. DLA Marbach 2. 12. 2015, abrufbar unter: https://www.netzliteratur.net/auer/Vom_Web_1.0_ zum_Postinternet.pdf (11. 3. 2021).

Auer, Johannes: worm applepie for doehl, 1997, abrufbar unter: https://auer.netzliteratur.net/ worm/applepie.htm (11. 3. 2021).

Bajohr, Hannes (Hrsg.) (2016): Code und Konzept. Literatur und das Digitale. Berlin: Frohmann Verlag.

Bourdieu, Pierre (1982): Die feinen Unterschiede. Kritik der gesellschaftlichen Urteilskraft. Frankfurt am Main: Suhrkamp.

Bourdieu, Pierre (1999): Die Regeln der Kunst. Genese und Struktur des literarischen Feldes. Frankfurt am Main: Suhrkamp.

Block, Friedrich [u.a.] (Hrsg.) (2004): p0es1s. Ästhetik digitaler Poesie. The Aesthetics of Digital Poetry. Ostfildern-Ruit: Hatje Cantz.

Goldsmith, Kenneth: Paragraphen zum konzeptuellen Schreiben, in: Edit, 63/2014.

Hartling, Florian (2009): Der digitale Autor. Autorschaft im Zeitalter des Internets. Bielefeld: Transcript Verlag.

Hausmann, Raoul (1972): Am Anfang war Dada. Frankfurt am Main: Anabas-Verlag Günter Kämpf, S. 76.

ORF.at: Jurydiskussion Jörg Piringer. 20. Juni 2020, abrufbar unter: https://bachmannpreis.orf. at/stories/3053821 (15. 3. 2021).

Piringer, Jörg: abcdefghijklmnopqrstuvwxyz, 2010, abrufbar unter: https://joerg.Piringer.net/index.php?href=abcdefg/abcdefg.xml (12. 3. 2021).

Piringer, Jörg [u.a.]: Das Branding besteht in der Individualität der einzelnen Bücher. In: etcetera Nr. 53 / LitArena 6 / Oktober 2013, abrufbar unter: https://www.litges.at/etcetera/interviews/j-Piringer-g-vallaster-das-branding-besteht-der-individualitat-der-einzelnen (11. 3. 2021). 
Piringer, Jörg (2018): datenpoesie. Klagenfurt: Ritterverlag.

Piringer, Jörg: fms, 2006. Video-Dokumentation abrufbar unter: https://vimeo.com/37308234 (11. 3. 2021).

Piringer, Jörg: Frikativ, 2009, abrufbar unter: https://joerg.Piringer.net/index.php?href=performance/frikativ.xml (11. 3. 2021).

Piringer, Jörg: kuzushi, 2020, abrufbar unter: https://files.orf.at/vietnam2/files/bachmannpreis/202019/kuzushi_jrg_Piringer_749200.pdf (15. 3. 2021).

Piringer, Jörg (2016): tiny poems. In Bajohr, Hannes (hrsg.): Code und Konzept. Literatur und das Digitale. Berlin: Frohmann Verlag.

Piringer, Jörg: was wird literatur? was wird poesie? In: Dossier: Was wird Literatur?, Literaturhaus Graz 9. November 2015, abrufbar unter: http://www.literaturhaus-graz.at/joerg-Piringer-waswird-literatur-was-wird-poesie (11. 3. 2020).

Reiche, Ruth (u. a.): Verfahren der Digital Humanities in den Geistes- und Kulturwissenschaften. Göttingen: GOEDOC, Dokumenten- und Publikationsserver der Georg-August-Universität, 2014, abrufbar unter: http://webdoc.sub.gwdg.de/pub/mon/dariah-de/dwp-2014-4.pdf (11. 3. 2021).

Stabauer, Annalena: Digitale Poesie. Hannes Bajohr: Halbzeug. Textverarbeitung. Jörg Piringer: datenpoesie. (2019 ): In: Der Hammer. Die Zeitung der Alten Schmiede, Nr. 103, S. 7.

Thalmair, Frank: Abstraktes Sprachgranulat. In: derStandard.at, 15.06.2009, abrufbar unter: http://derstandard.at/1242317325467/Joerg-Piringer-Abstraktes-Sprachgranulat (11. 3. 2021).

Vallaster, Günter: Jörg Piringer: datenpoesie. Literaturhaus Wien, 29. Oktober 2018, abrufbar unter: http:/ / www.literaturhaus.at/index.php?id=12197 (15. 3. 2021).

Wuchold, Cara: Digitale Poesie. Neue Dichtkunst mit dem Computer. In: Südwestrundfunk SWR2 Wissen, 9. Juni 2011, 8.30 Uhr, Redaktion: Anja Brockert, Regie: Günter Maurer. Sendemanuskript abrufbar unter: https://www.swr.de/-/id\%3D7985296/property\%3Ddownload/ nid\%3D660374/alnfcx/swr2-wissen-20110609.pdf (15. 3. 2021).

Dr. phil. Zdeněk Pecka / pecka@pf.jcu.cz

Jihočeská univerzita v Českých Budějovicích, Pedagogická fakluta, Katedra germanistiky Jeronýmova 10, 37005 České Budějovice, CZ 